\title{
Coexistence of Kondo effect and ferromagnetism in the Underscreened Kondo Lattice model
}

\author{
C. Thomas ${ }^{* \dagger}$, A. S. R. Simões*, J. R. Iglesias*, C. Lacroix ${ }^{\dagger}$, N. B. \\ Perkins** and B. Coqblin* \\ *Instituto de Física, Universidade Federal do Rio Grande do Sul, 91501-970 Porto Alegre, Brazil \\ ${ }^{\dagger}$ Institut Néel, CNRS-UJF, BP 166, 38042 Grenoble Cedex 9, France \\ ** Department of Physics, University of Wisconsin-Madison, Madison, WI 53706, USA \\ $\$$ Laboratoire de Physique des Solides, CNRS - Université Paris-Sud, 91405 Orsay, France
}

\begin{abstract}
In this work we use a Schrieffer-Wolff transformation in a two-fold degenerate periodic Anderson lattice to describe the coexistence of Kondo effect and ferromagnetism in some uranium and neptunium compounds. We show that the inclusion of a bandwidth for the $f$ electrons can account for a weak delocalization of $5 f$ electrons. Using a mean field approximation, we show that a maximum of $T_{C}$ versus $J_{K}$ can be found when the bandwidth is proportional to $J_{K}$.
\end{abstract}

Keywords: Kondo effect, ferromagnetism, Schrieffer-Wolff transformation

PACS: 71.27.+a, 75.30.Mb, 75.20.Hr, 75.10.-b

\section{INTRODUCTION}

The Kondo effect presents different aspects for $4 f$ and $5 f$ electrons. In the rareearth compounds, the $4 f$ electrons are well localized and can be described by the Doniach's diagram [1]. In the case of actinide compounds with $5 f$ electrons, it is known that some of them exhibit a coexistence of Kondo effect and ferromagnetism. Some uranium and neptunium compounds such as $\mathrm{UTe}[2,3,4], \mathrm{UCu}_{0.9} \mathrm{Sb}_{2}$ [5], $\mathrm{UCo}_{0.5} \mathrm{Sb}_{2}$ [6], $\mathrm{NpNiSi}_{2}$ [7] and $\mathrm{Np}_{2} \mathrm{PdGa}_{3}$ [8] present this behavior, with Curie temperatures of order $T_{C} \sim 50-100 \mathrm{~K}$, which are higher than the magnetic transition temperatures observed in the rare-earth compounds. Another interesting behavior is the variation of $T_{C}$ as a function of applied pressure in UTe $[9,10]$, where $T_{C}$ increases firstly with pressure, goes then through a maximum and finally decreases at very high pressures.

Perkins et al [11] proposed an underscreened Kondo Lattice model (UKL) to describe the coexistence of Kondo effect and ferromagnetism in the actinide compounds. The UKL model uses a complete localized description for the $5 f$ electrons. However, it is difficult to establish the degree of localization of $5 f$ electrons; for example, magnetic measurements in the uranium monochalcogenides show that US lies closest to the itinerant side for the $5 f$ electrons, USe is in the middle, and UTe presents more localized $5 f$ electrons [4].

Thus, we present here a review of our recent work [12], where we have shown that a delocalization of $5 f$ electrons can account for the variation of $T_{C}$ as a function of pressure. Starting from a two-fold degenerate periodic Anderson lattice, we use a Schrieffer-Wolff (SW) transformation [13] to obtain an effective bandwidth for the $f$ electrons. The dependence of the effective bandwidth appears to be very important for 
the variation of $T_{C}$ with the Kondo parameter $J_{K}$.

\section{EFFECTIVE MODEL}

To describe the coexistence between Kondo effect and ferromagnetism, we start from a periodic Anderson lattice model with two orbitals per site and $2 f$ electrons on these orbitals, which allows to treat the case of $S=1$ spins. The Hamiltonian can be written as:

$$
H=H_{s}+H_{f}+H_{\mathrm{hyb}},
$$

where $H_{s}$ is the kinetic energy of conduction electrons, $H_{f}$ represents the energy of $5 f$ electrons and $H_{\text {hyb }}$ is the hybridization between the conduction and $5 f$ electrons. Then,

$$
\begin{aligned}
H_{s}= & \sum_{\vec{k} \sigma} \varepsilon_{\vec{k}} c_{\vec{k} \sigma}^{\dagger} c_{\vec{k} \sigma}, \\
H_{f}= & \sum_{i \alpha \sigma} \varepsilon_{f} n_{i \alpha \sigma}^{f}+\sum_{i}\left[U\left(n_{i 1 \uparrow}^{f} n_{i 1 \downarrow}^{f}+n_{i 2 \uparrow}^{f} n_{i 2 \downarrow}^{f}\right)+U^{\prime}\left(n_{i 1 \uparrow}^{f} n_{i 2 \downarrow}^{f}+n_{i 1 \downarrow}^{f} n_{i 2 \uparrow}^{f}\right)\right. \\
& \left.+\left(U^{\prime}-J\right)\left(n_{i 1 \uparrow}^{f} n_{i 2 \uparrow}^{f}+n_{i 1 \downarrow}^{f} n_{i 2 \downarrow}^{f}\right)-J\left(f_{i 1 \uparrow}^{\dagger} f_{i 1 \downarrow} f_{i 2 \downarrow}^{\dagger} f_{i 2 \uparrow}+\text { h.c. }\right)\right], \\
H_{\mathrm{hyb}}= & \sum_{i \vec{k} \alpha \sigma}\left(V_{\vec{k} \alpha} e^{i \vec{k} \cdot \vec{R}_{i}} c_{\vec{k} \sigma}^{\dagger} f_{i \alpha \sigma}+V_{\vec{k} \alpha}^{*} e^{-i \vec{k} \cdot \vec{R}_{i}} f_{i \alpha \sigma}^{\dagger} c_{\vec{k} \sigma}\right),
\end{aligned}
$$

and we define

$$
H_{0}=H_{s}+H_{f}
$$

where $\varepsilon_{\vec{k}}$ is the energy of conduction electrons, $\varepsilon_{f}$ is the energy of the two-fold degenerate $f$ electrons, $U$ and $U^{\prime}$ are the Coulomb repulsion integrals between the $f$ electrons in the same and in different orbitals $\alpha(\alpha=1,2)$ and $J$ is the Hund's coupling constant. $c_{\vec{k} \sigma}^{\dagger}\left(c_{\vec{k} \sigma}\right)$ creates (destroys) a quasiparticle with momentum $\vec{k}$ and spin $\sigma, f_{i \alpha \sigma}^{\dagger}\left(f_{i \alpha \sigma}\right)$ is the creation (destruction) operator for $f$ electrons in the site $i$, orbital $\alpha$ and spin $\sigma$, and $n_{i \alpha \sigma}^{f}$ is the operator for the number of $f$ electrons.

To perform the SW transformation, we have to define the eigenstates of Hamiltonian $H_{0}$. We assume that the triplet states have lower energy comparing with the singlet states and they will be the groundstates. It means that we consider that the energy $U^{\prime}-J$ is much smaller than $U^{\prime}+J$ and $U$. We study the SW transformation in this limit, considering that just the triplet states, $S=1$, are initial and final states of the scattering process that give rise to the transformed Hamiltonian $H_{\text {trans }}=H_{0}+\tilde{H}$. The equation (6) describes the scattering process which plays the main role in the SW transformation [13]:

$$
\tilde{H}=\frac{1}{2} \sum_{c}\left\langle b\left|H_{\mathrm{hyb}}\right| c\right\rangle\left\langle c\left|H_{\mathrm{hyb}}\right| a\right\rangle\left(\frac{1}{E_{a}-E_{c}}+\frac{1}{E_{b}-E_{c}}\right)|b\rangle\langle a|,
$$

where $|a\rangle,|b\rangle$ and $|c\rangle$ are the eigenstates of $H_{0}$ and $E_{a}, E_{b}$ and $E_{c}$ are the eigenenergies, respectively. 
Among the different terms that come from the SW transformation, we obtain a Kondolike term that represents the interaction between the spins $S=1$ of localized $5 f$ electrons and the spins $s=1 / 2$ of conduction electrons and also a term that represents an effective bandwidth for the $f$ electrons.

From the SW transformation we obtain the $s-f$ interaction as:

$$
H_{S f}=\frac{1}{2} \sum_{\vec{i} \vec{k} k^{\prime}} J_{K}\left[c_{\overrightarrow{k^{\prime}} \uparrow}^{\dagger} c_{\vec{k} \downarrow} S_{i}^{f-}+c_{\overrightarrow{k^{\prime}} \downarrow}^{\dagger} c_{\vec{k} \uparrow} S_{i}^{f+}+\left(c_{\overrightarrow{k^{\prime}} \uparrow}^{\dagger} c_{\vec{k} \uparrow}-c_{\overrightarrow{k^{\prime}} \downarrow}^{\dagger} c_{\vec{k} \downarrow}\right) S_{z i}^{f}\right]
$$

where the operators $S_{i}^{f+}=n_{i 1}^{f} f_{i 2 \uparrow}^{\dagger} f_{i 2 \downarrow}+n_{i 2}^{f} f_{i 1 \uparrow}^{\dagger} f_{i 1 \downarrow}, S_{i}^{f-}=n_{i 1}^{f} f_{i 2 \downarrow}^{\dagger} f_{i 2 \uparrow}+n_{i 2 \sigma}^{f} f_{i 1 \downarrow}^{\dagger} f_{i 1 \uparrow}, S_{i}^{f z}=$ $n_{i 1 \uparrow}^{f} n_{i 2 \uparrow}^{f}-n_{i 1 \downarrow}^{f} n_{i 2 \downarrow}^{f}$ and $n_{i \alpha}^{f}=n_{i \alpha \uparrow}^{f}+n_{i \alpha \downarrow}^{f}$, satisfying the usual commutation rules for the spin operators. $J_{K}$ is the Kondo parameter and it is given by $J_{K}=\frac{2\left|V_{k f}\right|^{2}}{\mu-E_{0}^{f}}$, where $E_{0}^{f}=U^{\prime}-J+\varepsilon_{f}$ is the energy difference between one and two $f$ electrons with $S=1$ in the periodic Anderson model given by the equation (1). $\mu$ is the chemical potential and $V_{k f}$ is the Anderson hybridization.

The introduction of the $f$ bandwidth improves the decription of the $f$ electrons in the uranium and neptunium compounds. The effective $f$ bandwidth can be written in the mean field approximation as:

$$
A_{\vec{k} \sigma}=-P \frac{J_{K}}{2}\left(\left\langle n_{\sigma}^{f}\right\rangle^{2}+\frac{1}{2}\left\langle n_{\sigma}^{f}\right\rangle\left\langle n_{\bar{\sigma}}^{f}\right\rangle+\frac{1}{4}\left\langle n_{\bar{\sigma}}^{f}\right\rangle^{2}\right) \varepsilon_{\vec{k}}
$$

where $P$ is a parameter, $\left\langle n_{\sigma}^{f}\right\rangle$ is the mean value of the number of $f$ electrons per site and per orbital. In the original SW transformation for a localized spin $S=1 / 2$ lattice, an effective bandwidth term is also found. However, as the behavior of $4 f$ electrons are almost localized, this term is usually not considered. The effective Hamiltonian to be analyzed is:

$$
H_{\mathrm{eff}}=H_{0}+H_{s f}+\sum_{\vec{k} \alpha \sigma} A_{\vec{k} \sigma} f_{\vec{k} \alpha \sigma}^{\dagger} f_{\vec{k} \alpha \sigma}+\frac{1}{2} J_{H} \sum_{\langle i j\rangle} \vec{S}_{i}^{f} \cdot \vec{S}_{j}^{f} .
$$

The last term represents the RKKY interaction between the $f$ spins in different sites. As we want to study the coexistence of Kondo effect and ferromagnetism, this term is added to take into account the ferromagnetic interactions between the $f$ spins, where $J_{H}$ is a ferromagnetic interaction parameter. Thus in our model, both $J_{H}$ and the second order term in $J_{K}$ contribute to the determination of the Curie temperature.

\section{RESULTS AND CONCLUSION}

We have calculated the Kondo $\left(T_{K}\right)$ and Curie $\left(T_{C}\right)$ temperatures as a function of the Kondo parameter $J_{K}$. This allows us to obtain a comparison with the experimental results for the variation of $T_{K}$ and $T_{C}$ with applied pressure and our results present a 
good agreement with the experimental results for the compound UTe $[9,10] . T_{K}$ and $T_{C}$ are defined within the mean field approximation by the temperatures at which the magnetization and the mean value $\left\langle\lambda_{\sigma}\right\rangle=\left\langle c_{\vec{k} \sigma}^{\dagger} f_{i \alpha \sigma}\right\rangle$ are respectively tending to zero. We consider that the density of states for the conduction electrons is constant and equal to $2 D$. All energies are defined in units of $D$. We analyzed the effect of the $f$ bandwidth on the magnetic properties of the system, considering three different forms for the $f$ bandwidth, defined as $W$ :

- (a) $W$ is constant.

- (b) $W$ is proportional to the Kondo parameter, $W=Q J_{K}$.

- (c) $W$ is defined in agreement with our present result obtained by the SW transformation, $W=2 A_{\vec{k} \sigma} / \varepsilon_{\vec{k}}$.

In figure 1, we show the variation of $T_{C}$ and $T_{K}$ as a function of the Kondo parameter $J_{K}$ for the three cases, where we used $W=0.05$ for case (a), $Q=0.12$ for case (b), and $P=0.12$ for the case (c). For all figures we fixed the number of $f$ and $c$ electrons as $n_{\mathrm{tot}}^{f}=2$ and $n_{\mathrm{tot}}^{c}=0.8$, respectively.

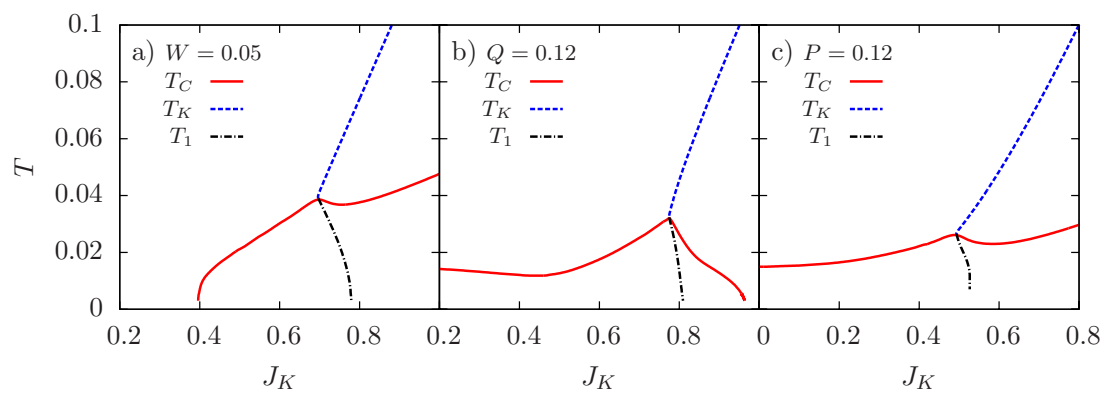

FIGURE 1. $T_{C}$ and $T_{K}$ versus $J_{K}$ for the three cases described in the text: (a) $W=0.05$, (b) $Q=0.12$, and (c) $P=0.12$, for $J_{H}=-0.01$ [12].

Cases (b) and (c), where the $f$ bandwidth is proportional to the Kondo parameter, show a maximum for $T_{C}$ for a given value of $J_{K}$ and a decrease of $T_{c}$ for larger $J_{K}$; the increase of $J_{K}$ can be connected with an increase of pressure, because, when the pressure increases, the energy $E_{0}^{f}$ comes closer to the Fermi level $\mu$, which results, as usual, in an increase of the value of $J_{K}$. We can conclude that the decrease of $T_{C}$ is related with the decrease of localization of $f$ electrons. This result is in agreement with the experimental results obtained for UTe $[9,10]$ and is in favor of the UKL model $[11,12]$ that we have proposed to describe the coexistence between Kondo effect and ferromagnetism in some uranium and neptunium compounds.

\section{ACKNOWLEDGMENTS}

This work was partially funded by Conselho Nacional de Desenvolvimento Científico e Tecnológico (CNPq) from Brazil 


\section{REFERENCES}

1. S. Doniach, Proceedings of the International Conference on Valence Instabilities and Related Narrow-band Phenomena, edited by R. D. Parks (Plenum Press, New Your, 1976), p. 168.

2. J. Schoenes, J. Less-Common Met., 121, 87 (1986).

3. J. Schoenes, B. Frick, and O. Vogt, Phys. Rev. B 30, 6578 (1984).

4. J. Schoenes, O. Vogt, J. Lohle, F. Hulliger, and K. Mattenberger, Phys. Rev. B 53, 14987 (1996).

5. Z. Bukowski, R. Troc, J. Stepien-Damm, C. Sulkowski, and V. H. Tran, J. Alloys Comp., 403, 65 (2005).

6. V. H. Tran, R. Troc, Z. Bukowski, D. Badurski, and C. Sulkowski, Phys. Rev. B 71, 094428 (2005).

7. E. Colineau, F. Wastin, J.P. Sanchez, and J. Rebizant, J. Phys.: Cond. Matter, 20, 075207 (2008).

8. V. H. Tran, J. C. Griveau, R. Eloirdi, W. Miiller, and E. Colineau, Phys. Rev. B 82, 094407 (2010).

9. A. L.Cornelius, J. S. Schilling, O. Vogt, K. Mattenberger, and U. Benedict, J. Magn. Magn. Mater. 161, 169 (1996).

10. P. Link, U. Benedict, J. Wittig, and H. Wühl, J. Phys.: Condens. Matter 4, 5585 (1992).

11. N. B. Perkins, M. D. Núñez-Regueiro, B. Coqblin, and J. R. Iglesias, Phys. Rev. B 76, 125101 (2007).

12. C. Thomas, A. S. R. Simões, J. R. Iglesias, C. Lacroix, N. B. Perkins, and B. Coqblin, Phys. Rev. B. 83, 014415 (2011)

13. J. R. Schrieffer and P. A. Wolff, Phys. Rev. 149, 491 (1966). 
Copyright of AIP Conference Proceedings is the property of American Institute of Physics and its content may not be copied or emailed to multiple sites or posted to a listserv without the copyright holder's express written permission. However, users may print, download, or email articles for individual use. 